\title{
Precursors of crystallization via density fluctuations in stiff-chain polymers
}

\author{
T. A. Ezquerra, ${ }^{1}$ E. López-Cabarcos, ${ }^{2}$ B. S. Hsiao, ${ }^{3}$ and F. J. Baltà-Calleja ${ }^{1}$ \\ ${ }^{1}$ Instituto de Estructura de la Materia, Consejo Superior de Investigaciones Científicas Serrano 119, Madrid 28006, Spain \\ ${ }^{2}$ Facultad de Farmacia, Universidad Complutense de Madrid, Madrid, Spain \\ ${ }^{3}$ Central Research and Development, E. I. DuPont de Nemours Experimental Station, Wilmington, Delaware 19880-0356
}

(Received 27 March 1995; revised manuscript received 8 February 1996)

\begin{abstract}
The ordering process just before cold crystallization for a stiff-chain polymer is studied by means of simultaneous real time wide- and small-angle x-ray scattering and dielectric spectroscopy techniques. The kinetics of the ordering process prior to crystallization is described by the spinodal decomposition formalism. From wide-angle $\mathrm{x}$-ray scattering measurements it is confirmed that no crystalline order takes place during the induction period. The dielectric study of the $\alpha$ relaxation suggests that during this induction period there is not a major restriction of segmental motions of polymer chain segments. [S1063-651X(96)02807-3]

PACS number(s): 82.35.+t, 61.10.Eq, 64.70.Pf
\end{abstract}

Long range density fluctuations of polymer chains have been proposed to be responsible for ordering phenomena occurring during the induction period prior to crystallization in poly(ethylene terephthalate) (PET) [1,2]. A parallel arrangement of polymer chains in given regions creates a density fluctuation which grows with time. Crystal nucleation occurs in the denser regions of the density fluctuation once the size of the fluctuation exceeds a given value $[3,4]$. This process is in agreement with the pioneering ideas of Flory according to which crystallization starts with a local parallelization of polymer chains, due to intramolecular interactions, followed by a subsequent increment in the intermolecular interaction between them [5]. The relative importance of these two steps is controlled by the chain flexibility. The ordered state prior to the crystalline one will be more stable than the disordered state provided that the chain flexibility is smaller than a critical value [5]. Recent calculations have shown that rigid rod polymers exhibit a critical concentration above which a nematic ordering is more stable than the isotropic one [6-8].

Parallelization prior to crystallization is a process whose kinetics has been tentatively described in terms of the spinodal decomposition formalism [1,2]. According to this view, in the early stages of phase separation the density fluctuation increases exponentially with time and the wave vector $(q$ $=(4 \pi / \lambda) \sin \theta)$ of the dominant fluctuation mode remains constant if the influence of thermal fluctuations is neglected [9-11]. In this case the phase separation can be analyzed in terms of Cahn-Hillard-Cook's linearized theory (CHC). In the late stages of spinodal decomposition, both the maximum scattered intensity $I_{m}$, and the most probable wave vector $q_{m}$ follow scaling laws of the type: $q_{m} \sim t^{-\alpha}$ and $I_{m} \sim t^{\gamma}[11-$ 13]. Moreover, spinodal decomposition has been observed in molten polymers submitted to force fields [14].

The aim of this paper is to provide evidence of density fluctuations, which can be considered precursors of crystal nucleation in the case of poly(ether ketone ketone) (PEKK) by using simultaneous real time small (SAXS) and wideangle x-ray scattering (WAXS) experiments. The advantage of simultaneous scattering measurements is the unambiguous assignment of the induction period prior to crystallization. PET contains ethylene groups in its chemical structure which suffer conformational changes upon heating above the glass transition temperature leading to a change of flexibility with temperature [1]. Therefore we have selected PEKK as a polymer system for this study, which has a higher chain stiffness than PET.

PEKK is a rigid polymer from the poly(aryl ether ketone) family which can be prepared with different para-meta $(T-I)$ phenyl isomer ratios [15]. In the present work $50 \mu \mathrm{m}$ amorphous 60-40 PEKK films, synthesized by DuPont, with a $T_{g}=428 \mathrm{~K}$ have been investigated. The sample preparation has been described elsewhere [16]. Simultaneous WAXS and SAXS experiments were performed using a double-focusing mirror monochromator camera on the polymer beam line at HASYLAB (Hamburg, F.R.G.). Cold crystallization was performed isothermally bringing the sample up to the crystallization temperature at a constant heating rate of $1{ }^{\circ} \mathrm{C} / \mathrm{min}$ from the glass state. Scattering patterns were recorded by using linear position-sensitive detectors and corrected for fluctuations in the intensity of the primary beam and background [16]. The data acquisition system is based on CAMAC hardware and modulator software [17]

Figures 1(a) and 1(b) show the variation of the wide- and small-angle x-ray scattering patterns as a function time for an isothermal experiment carried out at $T_{c}=183{ }^{\circ} \mathrm{C} \pm 0.5$. The patterns were obtained with an accumulation time of $120 \mathrm{~s}$. In the WAXS experiment the initial time pattern was subtracted from all the others in order to better observe the appearance of Bragg peaks indicating the growth of crystalline regions. The evolution of the SAXS patterns with time exhibits the appearance of a maximum of the scattered intensity at about $q=0.5 \mathrm{~nm}^{-1}$. The simultaneous WAXS experiment shows the onset of crystallization characterized by the appearance on Fig. 1(b) of two incipient maxima for times $t>2.4 \times 10^{3} \mathrm{~s}$ which are identified with the 110 and 200 reflections characteristic of a two chain orthorhombic unit cell $[16,15]$. A similar experiment was performed at $T=188^{\circ} \mathrm{C}$. The induction time in this case was $t \approx 1.2 \times 10^{3} \mathrm{~s}$.

The time evolution of the normalized integrated SAXS intensity $I_{\text {int }}(t)$ in the range $0.25 \mathrm{~nm}^{-1}<q<1 \mathrm{~nm}^{-1}$ at $188^{\circ} \mathrm{C}$ and $183^{\circ} \mathrm{C}$ is shown in Fig. 2. From the early times of the induction period for the isothermal process an increase of $I_{\text {int }}(t)$ is observed. For times $t>1.2 \times 10^{3}$ s at $T=188^{\circ} \mathrm{C}$ and $t>2.4 \times 10^{3} \mathrm{~s}$ at $183{ }^{\circ} \mathrm{C}$ (indicated by arrows in Fig. 2) 


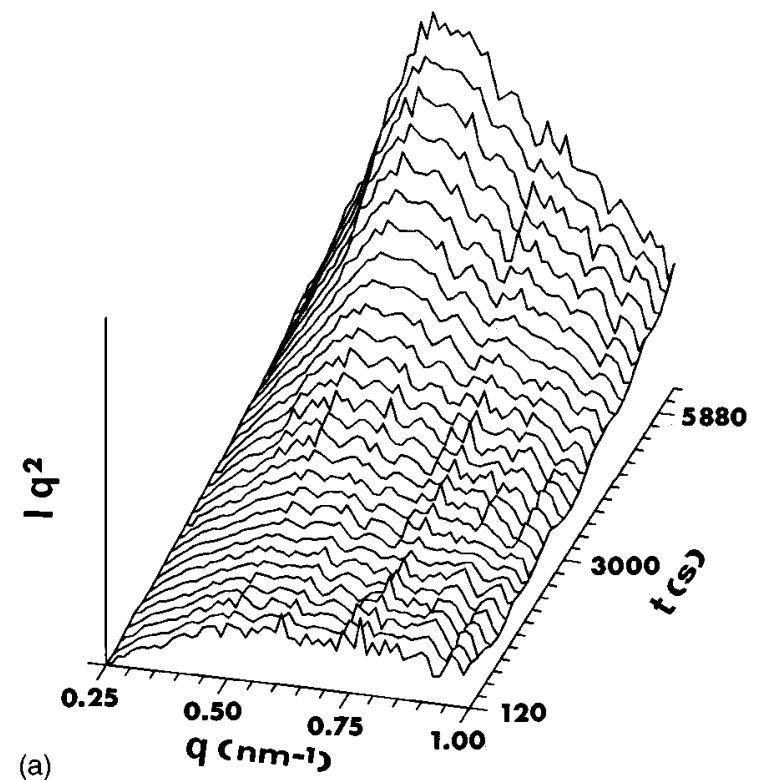

(a)

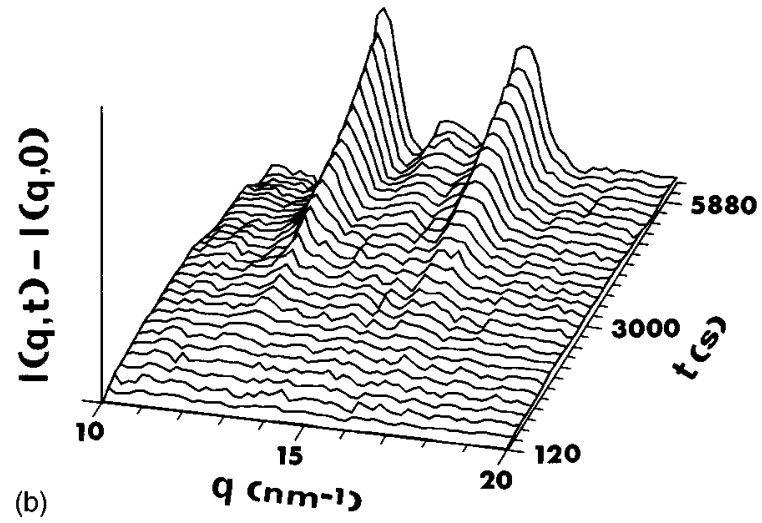

FIG. 1. Variation of (a) small- and (b) wide-angle $\mathrm{x}$-ray scattering during isothermal treatment of PEKK at $T=183{ }^{\circ} \mathrm{C} \pm 0.5$ from the glassy state. The diagrams were obtained simultaneously using synchrotron radiation.

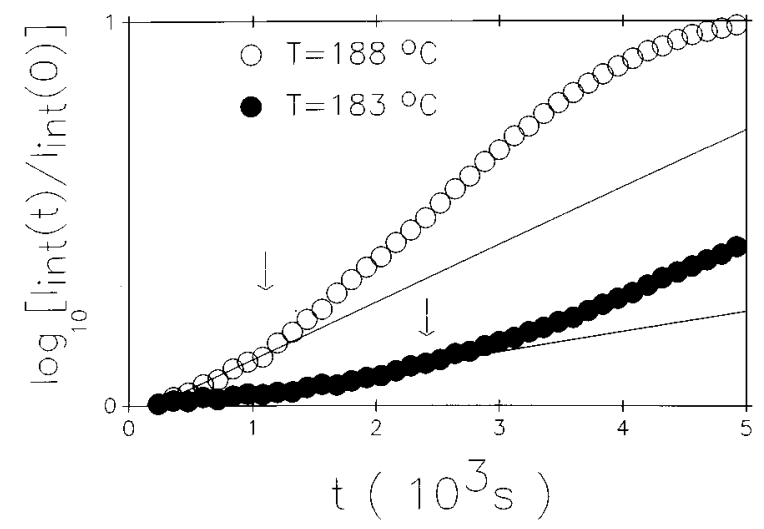

FIG. 2. Variation of $\log _{10}\left[I_{\text {int }}\right]$ as a function of time for different temperatures in the $q$ range $0.25 \mathrm{~nm}^{-1}<q<1 \mathrm{~nm}^{-1}$. The onset of crystallization, as measured from the real time WAXS patterns, is indicated by arrows.

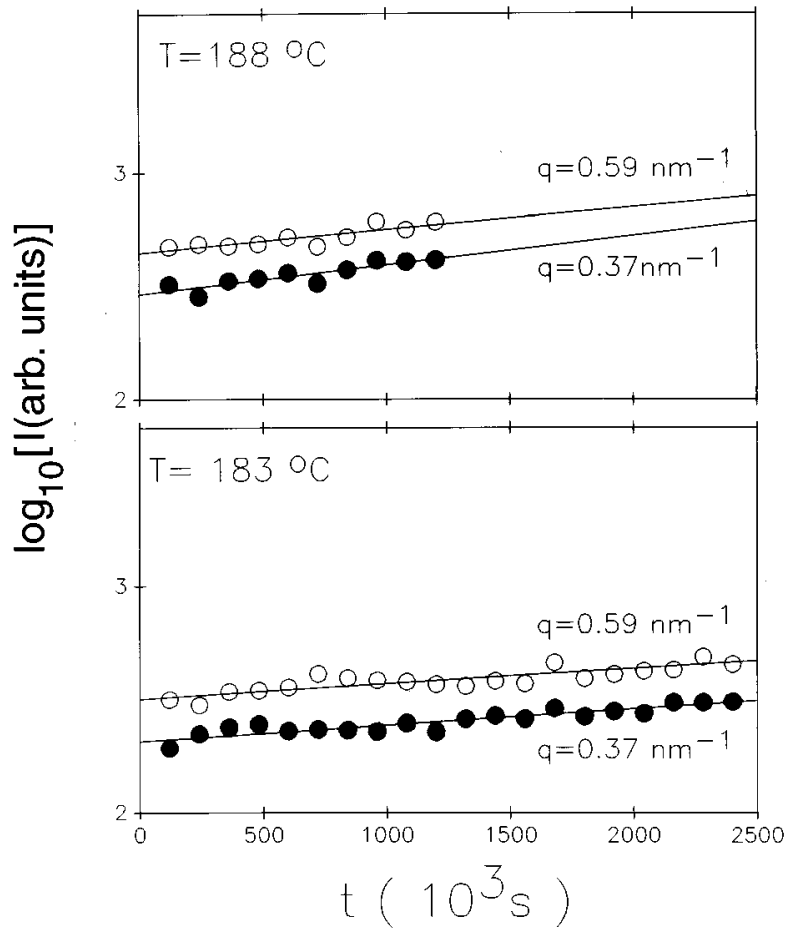

FIG. 3. Variation of $\log _{10}[I(q, t)]$ as a function of time for different values of $q$ at the two investigated temperatures.

the onset of crystallization is characterized by a greater increase of $I_{\text {int }}(t)$ concurrent with the appearance of Bragg reflexions in the WAXS experiment, which finally tend to level off for longer times.

During the early times of the isothermal process $I(q, t)$ increases exponentially with time as shown in Fig. 3. This time dependence agrees with the CHC's linearized theory for early stages of spinodal decomposition which predicts a variation of scattering intensity $I(q, t)$ as

$$
I(q, t)=I_{0} e^{2 R(q) t},
$$

being

$$
R(q)=D q^{2}\left[1-\frac{q^{2}}{2 q_{m}^{2}(0)}\right],
$$

where $I_{0}$ is the initial scattered intensity, $R(q)$ is the growth rate at the given $q, D$ is the apparent diffusion coefficient, and $q_{m}(0)$ is the initial most probable wavelength vector of fluctuation having the highest rate of growth. The values of the growth rate $R(q)$ can be calculated assuming the dependence predicted in Eq. (1). From the experimental data depicted in Fig. 3 values in the range $5 \times 10^{-4}$ $\mathrm{s}^{-1}>R(q)>5 \times 10^{-5} \mathrm{~s}^{-1}$ are obtained. The validity of the CHC linearized theory has been tested by plotting $R(q) / q^{2}$ versus $q^{2}$ as shown in Fig. 4. Here, a clear decrease of $R(q) / q^{2}$ with increasing $q^{2}$ is observed. From the extrapolation data in Fig. 4 to $q^{2}=0$ an estimation of $D=7 \times 10^{-4}$ $\mathrm{nm}^{2} / \mathrm{s}$ for $T=183{ }^{\circ} \mathrm{C}$ and $D=1.6 \times 10^{-3} \mathrm{~nm}^{2} / \mathrm{s}$ for $T=188^{\circ} \mathrm{C}$ can be extracted. These values are in the range of those expected for the apparent diffusion coefficient in polymeric systems [18]. From the intercept with the abscissa a characteristic $q$ value $q_{c}$ can be estimated from which the value 


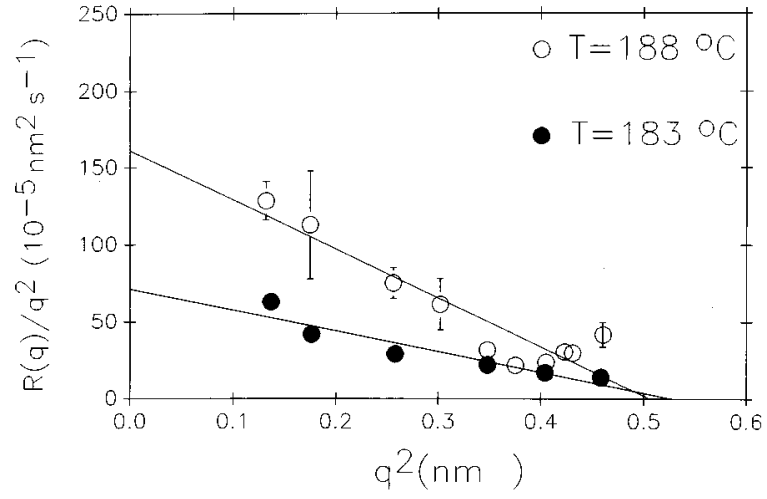

FIG. 4. Plot of $R(q) / q^{2}$ as a function of $q^{2}$ at the two investigated temperatures for the PEKK polymer.

corresponding to the most probable wave vector of fluctuation $q_{m}$ can be obtained according to the relation $q_{m}^{2}=q_{c}^{2} / 2$. In our case we obtain the result $q_{m}(0)=0.5 \mathrm{~nm}^{-1}$. It is noteworthy that this value is close to the observed $q_{\max }$ corresponding to the long period which appears in the final crystallization period. This result is different from that which has been observed in PET [19] where $q_{m}(0)$ appears well separated from the $q_{\max }$ corresponding to the long period. The different chain flexibility of PET and PEKK may be responsible for this fact. Moreover, from these results an estimation of the time for phase separation $\tau_{0}$ can be attempted considering that $\tau_{0}^{-1}=D q_{m}^{2}$. In our case $\tau_{0}$ values of $1.2 \times 10^{3} \mathrm{~s}$ and $2.8 \times 10^{3} \mathrm{~s}$ are obtained for $T=188^{\circ} \mathrm{C}$ and $T=183{ }^{\circ} \mathrm{C}$, respectively. As one may expect, the time for phase separation becomes longer at lower temperatures. According to these observations late stages of spinodal decomposition cannot be achieved due to the onset of crystallization for times close to $\tau_{0}$.

In order to assess independently the extension of the induction time in the 60-40 PEKK polymer, dielectric measurements under conditions nearly identical to those of the scattering experiments were performed. Figure 5 shows the $\alpha$ relaxation of PEKK (60-40) at $T=188^{\circ} \mathrm{C} \pm 1.5$ for $t=0$ and $t=30 \mathrm{~min}$. The $\alpha$ relaxation process is very sensitive to geometrical restrictions provoked by confinement of polymer chains in small pores [20] or by the presence of crystals [16]. These effects result in a retardation and broadening of the $\alpha$

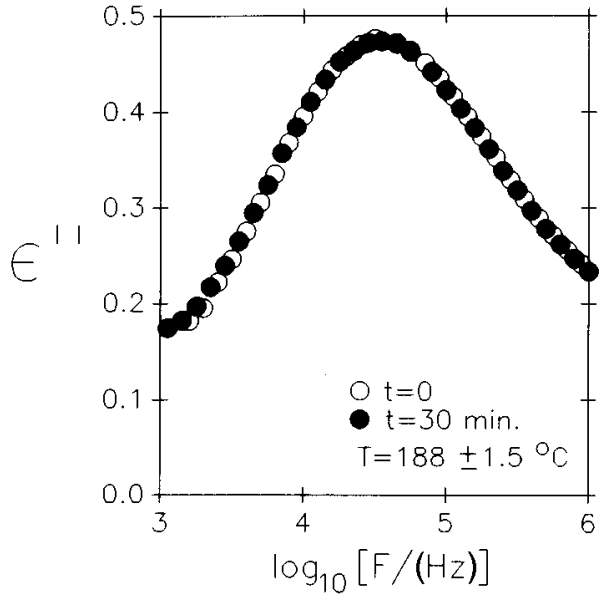

FIG. 5. Dielectric loss values versus frequency for PEKK (6040) at $T=188^{\circ} \mathrm{C} \pm 1.5$ for $(\bigcirc) t=0$ and $(\bigcirc) t=30 \mathrm{~min}$.

relaxation. According to Fig. 5, the invariance of the $\alpha$ relaxation, in our case after $t=30 \mathrm{~min}$, can be considered an indication that the microstructural changes occurring in this period are very localized and do not involve any significant restriction of the segmental mobility of the polymer chains.

In conclusion, the study of the ordering process, when amorphous PEKK is annealed above $T_{g}$, shows that a long range ordered structure with a size of about $12.5 \mathrm{~nm}^{-1}$ is formed during the induction period prior to crystallization. WAXS results confirm that no crystallization takes place during the induction period. The invariance of the $\alpha$ relaxation up to annealing times of $30 \mathrm{~min}$ suggests that microstructural changes in the induction period do not involve a severe restriction of segmental mobility of the polymer chains. The scattering behavior for the early stages before crystallization occurs can be described by Cahn-HillardCook's linearized theory of spinodal decomposition. Similar experiments in polymers with different chain flexibility are in progress.

The authors are indebted to the DGICYT (Grant No. PB 94-0049), Spain, and to NEDO's International Joint Research Program, Japan, for generous support of this investigation. Measurements at HASYLAB (Hamburg, F.R.G.) have been funded by the program Human Capital and Mobility, Access to Large Installations EC.
[1] M. Imai, K. Kaji, and T. Kanaya, Phys. Rev. Lett. 71, 4162 (1993).

[2] M. Imai, K. Mori, T. Mizukami, K. Kaji, and T. Kanaya, Polymer 33, 4451 (1992).

[3] B. Wunderlich, Macromolecular Physics (Academic New York, 1976), Vol. 1.

[4] K. F. Kelton, in Solid State Physics, edited by H. Ehrenreich and D. Turnbull (Academic, New York, 1991), Vol. 45, p. 75.

[5] P. J. Flory, Proc. R. Soc. London Ser. A 234, 60 (1956).

[6] T. Shimada, M. Doi, and K. Okano, J. Chem. Phys. 88, 2815 (1988).

[7] M. Doi, T. Shimada, and K. Okano, J. Chem. Phys. 88, 4070 (1988).
[8] M. Doi and S. F. Edwards, The Theory of Polymer Dynamics (Clarendon, Oxford, 1986).

[9] J. W. Cahn and J. H. Hilliard, J. Chem. Phys. 28, 258 (1958).

[10] H. E. Cook, Acta Metall. 18, 297 (1970).

[11] R. Bansil, J. Lal, and B. L. Carvalho, Polymer 33, 2961 (1992).

[12] K. Binder and D. Stauffer, Phys. Rev. Lett. 33, 1006 (1973).

[13] H. Furukawa, Adv. Phys. 34, 703 (1985).

[14] E. Brochard-Wyart and P. G. de Gennes, C. R. Acad. Sci. Ser. II 306, 699 (1988).

[15] K. H. Gardner, B. S. Hsiao, R. R. Matheson Jr., and B. A. Wood, Polymer 33, 2483 (1992).

[16] T. A. Ezquerra, J. Majszczyk, F. J. Baltá-Calleja, E. López- 
Cabarcos, K. H. Gardner, and B. S. Hsiao, Phys. Rev. B 50, 6023 (1994).

[17] C. Boulin, R. Kempf, M. H. J. Koch, and S. M. Mclaughlin, Nucl. Instrum. Methods Phys. Res. Sect. A 249, 399 (1986).

[18] T. P. Russell and I. Chin, Colloid Polym. Sci. 272, 1373
(1994).

[19] M. Imai, K. Kaji, T. Kanaya, and Y. Sakai, Phys. Rev. B 52, 12696 (1994).

[20] J. Schüller, Y. B. Mel'nichenko, R. Richert, and E. W. Fischer, Phys. Rev. Lett. 73, 2224 (1994). 